\title{
Symptomatic Urinary Tract Infections in Renal Transplant Recipients after Cystoscopy for Ureteral Stent Removal
}

\author{
Justin R. Gregg ${ }^{1,{ }^{*},}$ Caroline L. Kang ${ }^{1,{ }^{*}}$, Thomas R. Talbot ${ }^{2}$, Derek Moore ${ }^{3}$, S. Duke Herrell ${ }^{1}$, \\ Roger Dmochowski ${ }^{1}$, and Daniel A. Barocas ${ }^{1}$ \\ ${ }^{1}$ Vanderbilt University Medical Center, Department of Urologic Surgery, Nashville, TN \\ ${ }^{2}$ Vanderbilt University Medical Center, Department of Medicine, Nashville, TN \\ ${ }^{3}$ Vanderbilt University Medical Center, Department of Surgery, Nashville, TN
}

\section{Abstract}

Introduction-Symptomatic urinary tract infection (UTI) is a known complication of cystoscopy with ureteral stent removal. However, little is known about the incidence and risk factors for postcystoscopy UTI in renal transplant recipients, who likely represent a high-risk cohort. Our aim was to determine the infection rate following cystoscopy with stent removal in this population and identify opportunities for care improvement.

Methods-We performed a retrospective cohort study of office cystoscopies with stent removal in renal transplant recipients performed at a single institution from April 2012 through May 2014. Strict criteria were used to determine presence of symptomatic UTI within one month of the procedure. Fisher's exact tests were completed to examine associations between patient characteristics and post-transplant outcomes with UTI.

Results-A total of 324 patients were included. Mean age was 50.0 (SD 13.1) years, and 187 (57.7\%) patients were male. Within this group,165 (52.5\%) patients received a pre-procedural oral fluoroquinolone antibiotic dose prior to the procedure. Nine patients had symptomatic UTIs (2.8\%), of which three infections (33.3\%) were due to quinolone-resistant organisms. Female sex $(P=0.04)$, but no other patient or post-operative characteristic was associated with symptomatic UTI, including the use of peri-procedural antibiotics.

Conclusions-The incidence of symptomatic UTI after cystoscopy with ureteral stent removal in renal transplant recipients is less than three percent and comparable to post-cystoscopy UTI risk in the general population. Female sex is associated with symptomatic UTI. Further investigation is needed to identify groups most at risk for UTI and other complications.

\section{Keywords}

Cystoscopy; Ureteral catheterization; Renal transplantation; Urinary tract infection

*Corresponding author. Mailing address: Vanderbilt University Medical Center, 2525 West End, Suite 600, Nashville, TN 37203-1738. Phone: (615) 936-8322. Fax: (615) 343-8291. justin.r.gregg@ gmail.com.

Publisher's Disclaimer: This is a PDF file of an unedited manuscript that has been accepted for publication. As a service to our customers we are providing this early version of the manuscript. The manuscript will undergo copyediting, typesetting, and review of the resulting proof before it is published in its final citable form. Please note that during the production process errors may be discovered which could affect the content, and all legal disclaimers that apply to the journal pertain. 


\section{Introduction}

Cystoscopy for ureteral stent removal after renal transplant is a common reason for urology referral. Placement of stents after renal transplantation serves to decrease the occurrence of post-transplant urologic complications such as ureteral anastomotic leak and obstruction ${ }^{1,2}$ The transplant stent is typically removed by a urologist 4-6 weeks after transplant.

Urinary tract infection (UTI) is common after renal transplant, and evidence is conflicting regarding whether ureteral stent placement/removal further contributes to an increased incidence of bacteriuria or UTI. ${ }^{3,4}$ Due to the presence of comorbidities, recent genitourinary (GU) manipulation and the use of immunosuppressive medications to prevent organ rejection, renal transplant recipients are likely predisposed to acquiring infections and are commonly prescribed daily antibiotic prophylaxis. ${ }^{5}$

In the general population, cystoscopy is a well-tolerated procedure; however, urinary tract infection (UTI) is a known post-procedural complication. Studies report asymptomatic bacteriuria occurring in 5-25\% of patients and symptomatic infection occurring in approximately $5 \%$ of patients. ${ }^{6-9}$ Guidelines from the American Urological Association (AUA) and the American Society of Health-System Pharmacists (ASHP) recommend precystoscopy antibiotic administration in patients at increased risk of infection (e.g., advanced age, abnormal urinary tract, indwelling tubes, use of immunosuppression; Appendix) and those undergoing manipulation of their GU tract. ${ }^{10,11}$ Nonetheless, benefits of prophylaxis must always be balanced with risks of antibiotic complications and resistance. ${ }^{12}$

Prior studies have not examined the rate of infection after cystoscopy and stent removal when performed in renal transplant recipients. We performed a retrospective cohort study to determine the rate of symptomatic UTI in transplant patients undergoing cystoscopic stent removal, and the influence of antibiotic prophylaxis in this population.

\section{Methods}

\section{Clinic protocol}

Between April, 2012 and May, 2014, 5,488 outpatient cystoscopies were performed in the two outpatient urology clinics at our institution. Our cohort included all renal transplant patients who underwent outpatient cystoscopy with stent removal based on CPT and ICD-9 codes. All flexible cystoscopies were performed after genitalia were prepped with povidineiodine solution. $2 \%$ lidocaine jelly was instilled into the urethra prior to the procedure. Ureteral stent removal was performed using a flexible grasper, performed by an attending urologist (no stent had an attached string). After each procedure, cystoscopes were cleaned using an enzymatic cleanser (V. Mueller®), sterile water wash and chemical sterilization with paracetic acid (Steris $\mathrm{S} 40 \AA$ ). Following the procedure, patients were given an informational handout and instructed to notify the clinic if they developed a temperature $\geq 101^{\circ} \mathrm{F}$. Neither clinic had a formalized protocol regarding the use of pre-procedure prophylactic antibiotics, and administration of antibiotic prophylaxis was left to clinician 
preference. Patients were uniformly on prophylactic dose antibiotics (trimethoprimsulfamethoxazole or dapsone) prior to removal.

\section{Study Design}

After obtaining IRB approval, a retrospective review of medical records was performed and data was collected from electronic medical records. Data collected included patient characteristics (age, race, diabetes, smoking history, prednisone use, and deceased or living donor) and post-transplant outcomes as defined by patients' nephrologist (time to stent removal, readmission, non-genitourinary tract infection, delayed graft function, acute cellular rejection, and major urologic complication [urine leak]). Cases were defined as meeting the criteria for symptomatic UTI within 30 days after cystoscopy. Using prior literature, CDC surveillance definitions, and discussions with our hospital epidemiologist (TT), we developed the following criteria to define patients with symptomatic UTI ${ }^{13,14}$ :

1. One of the following signs or symptoms with no other recognized cause: fever, urgency, frequency, dysuria, prostate tenderness or suprapubic tenderness

AND

Positive urine culture ( $\geq 10^{5}$ microorganisms) with no more than two species present

OR

2. Two of the above signs and symptoms with no other recognized cause

AND

At least one of the following:

a. Positive dipstick for leukocyte esterase and/or nitrate

b. Pyuria (urine specimen with $>10$ white blood cell $[\mathrm{WBC}] / \mathrm{mm} 3$ )

c. Organisms seen on Gram's stain of unspun urine

d. At least two urine cultures with repeated isolation of the same uropathogen (gram negative bacteria or Staphylococcus saprophyticus) with $\geq 10^{2}$ colonies $/ \mathrm{mL}$ in nonvoided specimens

e. $<10^{5}$ colonies $/ \mathrm{mL}$ of a single uropathogen in a patient being treated with an effective antimicrobial agent for UTI

f. Physician diagnosis of UTI or prostatitis

g. Physician initiation of appropriate therapy for UTI or prostatitis

The student's t-test and Fisher's exact test were used to evaluate patient characteristics and transplant outcomes associated with symptomatic UTI. Chi-squared tests were two-sided and all tests with $P<0.05$ were considered significant. Statistics were completed using STATA (StataCorp, 2013 Stata Statistical Software: Release 13.1. College Station, TX). 
As a secondary outcome, we investigated whether the use of prophylactic antibiotics, in addition to daily prophylaxis taken by all transplant patients at our institution for prevention of infectious diseases, was associated with post-procedural symptomatic UTI.

Prior studies indicate that patients undergoing cystoscopy at our institution receive prophylactic antibiotics approximately $75 \%$ of the time. ${ }^{15}$ With a study cohort of approximately 320 transplant patients, we calculated that we had $>80 \%$ power to detect a difference in symptomatic UTI of $2 \%$ in those who received antibiotics vs. $10 \%$ in those who did not, with a 0.05 type I error probability. Given that transplant patients are already on a daily antibiotic and may receive less prophylactic dosing, we calculate that power approaches $98 \%$ if half of the cohort receives prophylactic antibiotics.

\section{Results}

A total of 324 renal transplant patients underwent cystoscopy with stent removal during the study time period. Patient characteristics are shown in Table 1. Average age was 50 years. $42.3 \%$ of patients were female and $70.4 \%$ were white. Stents were removed an average of 28.6 days (SD 6.9) after transplant, and timing was left to the discretion of the referring transplant team. All patients were taking trimethoprim/sulfamethoxazole or dapsone as prophylactic antibiotics. An additional $165(52.5 \%)$ patients received a dose of prophylactic antibiotic prior to cystoscopy to remove ureteral stents. Of these, 163/165 (98.8\%) received a fluoroquinolone as pre-procedural prophylaxis. No adverse events related to antibiotic use were noted in patients who received a prophylactic fluoroquinolone.

Nine (2.8\%) patients met criteria for symptomatic UTI within 30 days after cystoscopy. Eight of the 9 symptomatic UTIs had a positive urine culture, of which the involved bacteria were Escherichia coli $(\mathrm{N}=3)$, Klebsiella pneumoniae $(\mathrm{N}=2)$, Citrobacter (2 different species) and Enterococcus faecalis $(\mathrm{N}=1)$. The one patient with culture negative urine had a UTI diagnosed by a physician due to frequency, incomplete emptying and prostatic tenderness. Three (37.5\%) of the 8 bacterial isolates were resistant to fluoroquinolones. Two of the 9 patients with UTI required admission for treatment of pyelonephritis. None required ICU admission. Within the cohort, 21 patients $(6.5 \%)$ had asymptomatic bacteriuria.

Table 2 shows patient characteristic and post-transplant measures and their association with symptomatic UTI. Female sex was associated with symptomatic UTI ( $\mathrm{p}=0.04)$. No other patient or transplant-related factor was associated with UTI, though and lack of prednisone as an immunosuppressive agent $(P=0.10)$ trended toward association with symptomatic UTI. Notably, lack of prophylactic antibiotic use prior to cystoscopy with stent removal was not associated with symptomatic UTI ( $\mathrm{p}=0.32$, Figure).

\section{Discussion}

In this cohort of renal transplant patients undergoing cystoscopic ureteral stent removal, we found a $2.8 \%$ rate of symptomatic UTI. Thus, despite multiple factors that may place this group at an increased risk of UTI, including the presence of comorbidities, use of immunosuppressive medications and recent surgical intervention, ${ }^{13}$ the risk of UTI after 
stent removal in renal transplant patients may not be different from that found in the general population.

The low rate of symptomatic UTI seen in our cohort differs from that seen in many studies examining infections in the post-renal transplant population. While the majority of these studies examine patients for at least three months after transplant (as opposed to one month post-stent removal in our cohort), the rates of infection are strikingly different. Large studies examining only acute graft pyelonephritis (fever, positive urine culture and urinary symptoms) have shown rates of infection at $13-16.5 \% .{ }^{16,17}$ This contrasts with the two out of $324(0.6 \%)$ patients who had acute pyelonephritis in our study, albeit with admittedly shorter observation time. In terms of cystitis, three retrospective cohorts of over 100 patients each demonstrated UTI rates of $34.5 \%,{ }^{18} 44.8 \%{ }^{19}$ and $33.7 \%,{ }^{20}$ respectively. Importantly, these studies included patients with asymptomatic positive urine culture results in their definition of UTI, representing much less stringent criteria. While some believe that asymptomatic bacteriuria is a reasonable outcome in a transplant population given a possible association with graft related complications, ${ }^{21}$ evidence is lacking as to whether intervention on asymptomatic bacteriuria has any effect on transplant-related outcomes. Furthermore, the role of daily low-dose prophylactic antibiotics on bacteriuria was not established at the time of these studies, as evidenced by the $6.5 \%$ rate of asymptomatic bacteriuria seen in our population, among which all patients received daily prophylaxis.

Multiple randomized prospective studies investigating outpatient flexible cystoscopy have demonstrated low rates of UTI in patients randomized to pre-procedural placebo administration. While definition of UTI varied, recent large studies demonstrated symptomatic UTI rates of $3 \%,{ }^{22} 2.5 \%,{ }^{23}$ and $0.8 \% .{ }^{24}$ Notably, patients within these studies did not undergo cystoscopy with urinary tract manipulation such as stent removal. Guidelines place patients at increased risk for infection when manipulation occurs, even though evidence supporting this practice has largely been extrapolated from studies investigating the efficacy of prophylactic antibiotic use prior to trans-urethral resection of the prostate. ${ }^{10} \mathrm{~A}$ number of prior studies, while limited by the above-mentioned broad definition of UTI, have investigated risk factors associated with infection in patients after renal transplant. A retrospective analysis by Camargo et al. demonstrated that older age, acute rejection and deceased donor transplant were associated with UTI (definition unknown, though likely included bacteriuria given a 33.7\% rate of infection). A separate cohort showed that immunosuppressive regimen, deceased donor transplant, duration of urinary bladder catheterization and length of hospital stay were associated with symptomatic UTI or bacteriuria. ${ }^{19}$ In our contemporary cohort, female sex $(P=0.04)$ was associated with symptomatic UTI. This finding is likely valid, as female sex is a known risk factor for UTI, ${ }^{14}$ and is mechanistically plausible given the short female urethral length. Further studies are needed, particularly to determine if antibiotics may be safely omitted in select renal transplant patients.

Antibiotics implicitly risk complications such as gastrointestinal distress, opportunistic infections and drug reactions. ${ }^{25}$ These potential downsides, as well as increasing rates of antimicrobial resistance, ${ }^{26,14}$ have contributed to an increase in awareness of the need for urologists to practice antibiotic stewardship. ${ }^{27}$ Furthermore, a recent study advocates for 
consideration of prophylactic antibiotic omission in patients undergoing outpatient cystoscopy, including those who are at increased risk for infection. ${ }^{6}$ While infectious disease ${ }^{11}$ and urology ${ }^{10}$ guidelines do not advocate for such an approach, a recent study by our group demonstrated that more narrow criteria may be safely used to risk stratify patients undergoing outpatient cystoscopy, especially given high rates of resistance to recommended prophylactics. ${ }^{15}$ Interestingly, treatment dose antibiotic prophylaxis was not associated with UTI in this cohort, a group with multiple risk factors for UTI as defined by the aforementioned guidelines, including immune suppression, recent hospitalization and GU tract manipulation.

Given this finding, it may be reasonable to advocate for decreased peri-procedural antibiotic use in this population, especially given the broad use of daily antibiotic prophylaxis in this group. However, a difference in absolute rates of symptomatic UTI was demonstrated in our cohort, with $3.9 \%$ of patients in whom prophylaxis was omitted having a symptomatic UTI, compared to $1.8 \%$ of those who received pre-procedural medication. These rates of UTI are actually lower than those expected at the onset of the study. A post-hoc power calculation reveals that 640 patients would have been needed to detect a significant difference given the rates of infection and pre-procedural antibiotic use in our cohort. Our study was therefore limited in sample size and the finding that antibiotic prophylaxis is not associated with symptomatic UTI may reflect a type II error. Other limitations of our study, including potential bias related to its retrospective nature which may have limited the number of identified UTIs, make it difficult to definitively conclude that peri-procedural antibiotics do not prevent symptomatic UTI in this population. Additionally, pre-procedural urine cultures were not uniformly available to guide prophylaxis. Given the potential consequences of a severe urinary tract infection in this population, including complications related to graft health or immune suppression, we do not recommend cessation of prophylactic antibiotic dosing in this group until larger, prospective studies have been completed.

To our knowledge, this is the first study to determine the rate of symptomatic UTI after postrenal transplant outpatient cystoscopy and stent pull. The infection rate of $2.8 \%$ is comparable to rates of post-cystoscopy symptomatic UTI in the general population, suggesting that UTI risk in this group may be lower than previously expected. We additionally identified sex as a factor associated with symptomatic UTI after stent removal. While the efficacy of full-dose prophylactic antibiotics in prevention of post-procedural UTI remains to be proven, transplant patients represent an at-risk population for which preprocedural antibiotic prophylaxis should likely continue. Further prospective studies (ideally in the setting of a randomized clinical trial) are needed to determine patients most at risk for symptomatic UTI after renal transplant.

\section{Acknowledgments}

Funding Support

Data collection and analysis were supported in part by National Center for Advancing Translational Sciences/ National institutes of Health grant UL1 TR000445. 


\title{
Key of Definitions and Abbreviations
}

\author{
ASHP American Society of Health-System Pharmacists \\ AUA American Urological Association \\ GU Genitourinary \\ UTI Urinary Tract Infection
}

\section{Works Cited}

1. Harza M, Baston C, Preda A, et al. Impact of ureteral stenting on urological complications after kidney transplantation surgery: a single-center experience. Transplant Proc. 2014; 46:3459-3462. [PubMed: 25498072]

2. Wilson CH, Rix DA, Manas DM. Routine intraoperative ureteric stenting for kidney transplant recipients. Cochrane Database Syst Rev. 2013; 6:CD004925.

3. Chordia P, Schain D, Kayler L. Effects of ureteral stents on risk of bacteriuria in renal allograft recipients. Transpl Infect Dis. 2013; 15:268-275. [PubMed: 23421817]

4. Galindo Sacristán P, Pérez Marfil A, Osorio Moratalla JM, et al. Predictive factors of infection in the first year after kidney transplantation. Transplant Proc. 2013; 45:3620-3623. [PubMed: 24314976]

5. Green H, Rahamimov R, Gafter U, et al. Antibiotic prophylaxis for urinary tract infections in renal transplant recipients: a systematic review and meta-analysis. Transpl Infect Dis. 2011; 13:441-447. [PubMed: 21521435]

6. Herr HW. Should Antibiotics Be Given Prior to Outpatient Cystoscopy? A Plea to Urologists to Practice Antibiotic Stewardship. Eur Urol. 2014; 65:839-842. [PubMed: 24012206]

7. Rané A, Cahill D, Saleemi A, et al. The issue of prophylactic antibiotics prior to flexible cystoscopy. Eur Urol. 2001; 39:212-214. [PubMed: 11223682]

8. Clark KR, Higgs MJ. Urinary infection following out-patient flexible cystoscopy. Br J Urol. 1990; 66:503-505. [PubMed: 2249120]

9. Alsaywid B, Smith GH. Antibiotic prophylaxis for transurethral urological surgeries: Systematic review. Urol Ann. 2013; 5:61. [PubMed: 23798859]

10. Wolf JS Jr, Bennett CJ, Dmochowski RR, et al. Best Practice Policy Statement on Urologic Surgery Antimicrobial Prophylaxis. J Urol. 2008; 179:1379-1390. [PubMed: 18280509]

11. Bratzler DW, Dellinger EP, Olsen KM, et al. Clinical practice guidelines for antimicrobial prophylaxis in surgery. Am J Health Syst Pharm. 2013; 70:195-283. [PubMed: 23327981]

12. Bootsma AMJ, Laguna Pes MP, Geerlings SE, et al. Antibiotic Prophylaxis in Urologic Procedures: A Systematic Review. Eur Urol. 2008; 54:1270-1286. [PubMed: 18423974]

13. Gould CV, Umscheid CA, Agarwal RK, et al. Guideline for prevention of catheter-associated urinary tract infections 2009. Infect Control Hosp Epidemiol. 2010; 31:319-326. [PubMed: 20156062]

14. Pollack LA, Srinivasan A. Core Elements of Hospital Antibiotic Stewardship Programs From the Centers for Disease Control and Prevention. Clin Infect Dis. 2014; 59:S97-S100. [PubMed: 25261548]

15. Gregg JR, Lai C, Dmochowski R, et al. Recent Antibiotic Treatment Increases the Risk of PostOutpatient Cystoscopy Urinary Tract Infection. Urol Pract. 2015

16. Giral M, Pascuariello G, Karam G, et al. Acute graft pyelonephritis and long-term kidney allograft outcome. Kidney Int. 2002; 61:1880-1886. [PubMed: 11967040]

17. Kamath NS, John GT, Neelakantan N, et al. Acute graft pyelonephritis following renal transplantation. Transpl Infect Dis Off J Transplant Soc. 2006; 8:140-147.

18. Veroux M, Giuffrida G, Corona D, et al. Infective complications in renal allograft recipients: epidemiology and outcome. Transplant Proc. 2008; 40:1873-1876. [PubMed: 18675076] 
19. Dantas SRPE, Kuboyama RH, Mazzali M, et al. Nosocomial infections in renal transplant patients: risk factors and treatment implications associated with urinary tract and surgical site infections. J Hosp Infect. 2006; 63:117-123. [PubMed: 16517007]

20. Camargo LF, Esteves ABA, Ulisses LRS, et al. Urinary Tract Infection in Renal Transplant Recipients: Incidence, Risk Factors, and Impact on Graft Function. Transplant Proc. 2014; 46:1757-1759. [PubMed: 25131029]

21. Yacoub R, Akl NK. Urinary tract infections and asymptomatic bacteriuria in renal transplant recipients. J Glob Infect Dis. 2011; 3:383-389. [PubMed: 22224004]

22. García-Perdomo HA, López H, Carbonell J, et al. Efficacy of antibiotic prophylaxis in patients undergoing cystoscopy: a randomized clinical trial. World J Urol. 2013; 31:1433-1439. [PubMed: 23412704]

23. Johnson MI, Merrilees D, Robson WA, et al. Oral ciprofloxacin or trimethoprim reduces bacteriuria after flexible cystoscopy. BJU Int. 2007; 100:826-829. [PubMed: 17822463]

24. Wilson L, Ryan J, Thelning C, et al. Is Antibiotic Prophylaxis Required for Flexible Cystoscopy? A Truncated Randomized Double-Blind Controlled Trial. J Endourol. 2005; 19:1006-1008. [PubMed: 16253070]

25. Schaeffer, A., Schaeffer, E. Campbell-Walsh Urology. 10. Philadelphia, PA: Elsevier Saunders; 2012. Infecions of the Urinary Tract; p. 2335-2354.

26. Sanchez GV, Master RN, Karlowsky JA, et al. In vitro antimicrobial resistance of urinary Escherichia coli isolates among U.S. outpatients from 2000 to 2010. Antimicrob Agents Chemother. 2012; 56:2181-2183. [PubMed: 22252813]

27. Coleman S, Goldman HB. Antibiotic Stewardship in Urology. Urol Pract. 2015; 2:270-274.

\section{Appendix. Published Risk Factors for Infection Post-Cystoscopy}

\begin{tabular}{|c|c|}
\hline $\mathbf{A U A}^{*}$ & ASHP $^{* * *}$ \\
\hline Advanced Age & Extremes of age \\
\hline Poor nutritional status & Nutritional status \\
\hline Smoking & Tobacco use \\
\hline Distant coexistent infection & Coexistent remote body-site infection \\
\hline Immunodeficiency & Altered Immune Response \\
\hline Chronic corticosteroid use & Corticosteroid Therapy \\
\hline Prolonged hospitalization & Length of Preoperative Hospitalization \\
\hline Colonized endogenous or exogenous material & Colonization with Microorganisms \\
\hline \multirow[t]{7}{*}{ Anatomic anomalies of the urinary tract } & Anatomic Anomalies of the Urinary Tract \\
\hline & Obesity \\
\hline & Diabetes mellitus \\
\hline & Recent Surgical Procedure \\
\hline & Urinary Obstruction \\
\hline & Urinary Stone \\
\hline & Indwelling or Externalized Catheter \\
\hline
\end{tabular}




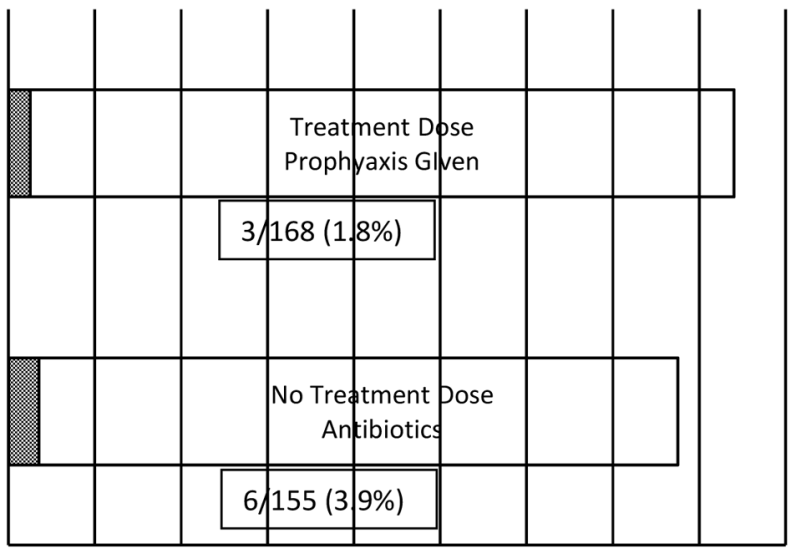

四TI

口No UTI

Number of patients

\section{$\mathbf{P}=\mathbf{0 . 4 7}$}

Figure 1.

Effect of Pre-Cystoscopy Full-Dose Dose Antibiotic Prophylaxis on Symptomatic UTI. Medical records of renal transplant patients who underwent cystoscopy for stent removal were examined for pre-cystoscopy antibiotic administration and symptomatic UTI criteria 30 days after cystoscopy. Patients who met criteria for UTI are represented in gray and patients who did not meet criteria for UTI were represented in white. Patients who did and did not receive pre-cystoscopy treatment dose prophylaxis in addition to universally administered daily prophylaxis are shown in the upper and lower bars, respectively. 
Table 1

\section{Patient Characteristics}

\begin{tabular}{|c|c|}
\hline Characteristic & Number $(\%)$ \\
\hline Age (mean [STD]) & 50.0 \\
\hline \multicolumn{2}{|l|}{ Sex } \\
\hline Male & $187(57.7)$ \\
\hline Female & $137(42.3)$ \\
\hline \multicolumn{2}{|l|}{ Race } \\
\hline White & $228(70.4)$ \\
\hline Black & $88(27.2)$ \\
\hline Hispanic & $2(0.62)$ \\
\hline Asian & $6(1.9)$ \\
\hline \multicolumn{2}{|l|}{ Diabetes mellitus } \\
\hline No & $220(67.9)$ \\
\hline Yes & $104(32.1)$ \\
\hline \multicolumn{2}{|l|}{ Smoking history } \\
\hline No & $231(71.3)$ \\
\hline Yes & $93(28.7)$ \\
\hline \multicolumn{2}{|l|}{ Prednisone } \\
\hline No & $158(48.8)$ \\
\hline Yes & $166(51.2)$ \\
\hline \multicolumn{2}{|l|}{ Donor kidney type } \\
\hline Living & $114(35.2)$ \\
\hline Deceased & $210(64.8)$ \\
\hline \multicolumn{2}{|c|}{ Time from transplant to stent removal } \\
\hline$<4$ weeks & $199(61.4)$ \\
\hline$>4$ weeks & $125(38.6)$ \\
\hline \multicolumn{2}{|c|}{ Received preprocedure antibiotics } \\
\hline No & $156(48.1)$ \\
\hline Yes & $168(51.9)$ \\
\hline \multicolumn{2}{|c|}{ Readmission to the hospital one month prior to cystoscopy } \\
\hline No & $233(71.9)$ \\
\hline Yes & $91(28.1)$ \\
\hline \multicolumn{2}{|c|}{ Non-genitourinary infection one month prior to cystoscopy } \\
\hline No & $294(90.7)$ \\
\hline Yes & $30(9.3)$ \\
\hline \multicolumn{2}{|l|}{ Delayed graft function } \\
\hline No & $216(66.7)$ \\
\hline Yes & $108(33.3)$ \\
\hline Acute transplant rejection & \\
\hline
\end{tabular}

Urol Pract. Author manuscript; available in PMC 2018 September 01. 


\begin{tabular}{|l|c|}
\hline Characteristic & Number (\%) \\
\hline No & $304(93.8)$ \\
\hline Yes & $20(6.2)$ \\
\hline Major urological complication & \\
\hline No & $320(98.8)$ \\
\hline Yes & $4(1.2)$ \\
\hline
\end{tabular}


Table 2

Post-transplant and Patient Characteristics Associated with Post-Cystoscopy UTI

\begin{tabular}{|c|c|c|c|}
\hline \multirow{2}{*}{ Characteristic } & \multicolumn{2}{|c|}{ UTI } & \multirow{2}{*}{$P$-value } \\
\hline & No & Yes & \\
\hline Age (mean [STD]) & $50.0(13.0)$ & $49.5(18.0)$ & 0.45 \\
\hline Sex & & & 0.04 \\
\hline Male & $185(58.7)$ & $2(22.2)$ & \\
\hline Female & $130(41.3)$ & $7(77.8)$ & \\
\hline Race & & & 0.73 \\
\hline Caucasian & $222(70.5)$ & $4(44.4)$ & \\
\hline Other & $99(29.5)$ & $5(55.6)$ & \\
\hline Diabetes mellitus & & & 0.15 \\
\hline No & $216(68.6)$ & $4(44.4)$ & \\
\hline Yes & $99(31.4)$ & $5(55.67)$ & \\
\hline Smoking history & & & 0.72 \\
\hline No & $225(71.4)$ & $6(66.7)$ & \\
\hline Yes & $90(28.6)$ & $3(33.3)$ & \\
\hline Prednisone & & & 0.10 \\
\hline No & $150(47.6)$ & $7(77.8)$ & \\
\hline Yes & $165(52.4)$ & $2(22.2)$ & \\
\hline Donor kidney type & & & 0.50 \\
\hline Living & $112(35.6)$ & $2(22.2)$ & \\
\hline Deceased & $203(64.4)$ & $7(77.8)$ & \\
\hline Time from transplant to stent removal & & & 1.0 \\
\hline$<4$ weeks & $194(61.6)$ & $6(66.7)$ & \\
\hline$>4$ weeks & $121(38.4)$ & $3(33.3)$ & \\
\hline Received preprocedure antibiotics & & & 0.32 \\
\hline No & $149(47.5)$ & $6(66.7)$ & \\
\hline Yes & $165(52.5)$ & $3(33.3)$ & \\
\hline Hospitalized within one month of cystoscopy & & & 0.28 \\
\hline No & $228(72.4)$ & $5(55.6)$ & \\
\hline Yes & $87(27.6)$ & $4(44.4)$ & \\
\hline Non-genitourinary infection one month prior to cystoscopy & & & 0.20 \\
\hline No & $287(91.1)$ & $7(77.8)$ & \\
\hline Yes & $28(8.9)$ & $2(22.2)$ & \\
\hline
\end{tabular}

